\title{
BMJ Open Seroprevalence of SARS-CoV-2 in the West Bank region of Palestine: a cross- sectional seroepidemiological study
}

\author{
Nouar Qutob (D) , Faisal Awartani, Zaidoun Salah, Mohammad Asia, \\ Imad Abu Khader, Khaled Herzallah, Nadeen Balqis, Husam Sallam
}

To cite: Qutob N, Awartani F, Salah Z, et al. Seroprevalence of SARS-CoV-2 in the West Bank region of Palestine: a crosssectional seroepidemiological study. BMJ Open

2021;11:e044552. doi:10.1136/ bmjopen-2020-044552

- Prepublication history and additional material for this paper are available online. To view these files, please visit the journal online (http://dx.doi. org/10.1136/bmjopen-2020044552).

Received 17 September 2020 Revised 06 January 2021 Accepted 07 January 2021

Check for updates

(C) Author(s) (or their employer(s)) 2021. Re-use permitted under CC BY-NC. No commercial re-use. See rights and permissions. Published by BMJ.

Department of Health Sciences, Faculty of Graduate Studies, Arab American University, Ramallah, Palestine

Correspondence to

Dr Nouar Qutob;

nouar.qutob@aaup.edu

\section{ABSTRACT}

Objectives Seroprevalence rates are important indicators to the epidemiology of COVID-19 and the extent of the pandemic given the existence of asymptomatic cases. The purpose of this study is to assess the seroprevalence rate in the Palestinian population residing in the West Bank. Setting The study involved 1355 participants from 11 governorates, including 112 localities in the West Bank and 1136 individuals visiting Palestinian medical laboratories. Participants Blood samples were collected between 15th June 2020 and 30th June 2020 from 1355 individuals from randomly selected households in the West Bank, in addition to 1136 individuals visiting Palestinian medical laboratories between the 1st May 2020 and 9th July 2020 for a routine check-up.

Primary and secondary outcome measures Out of the 2491 blood samples collected, serological tests for 2455 adequate serum samples were done using an immunoassay for qualitative detection of antibodies against SARS-CoV-2. Seroprevalence was estimated as the proportion of individuals who had a positive result in the total SARS-CoV-2 antibodies in the immunoassay.

Results The random sample of Palestinians living in the West Bank yielded 0\% seroprevalence with $95 \%$ and an adjusted $\mathrm{Cl}(0 \%$ to $0.0043 \%)$, while the lab referral samples yielded an estimated seroprevalence of $0.354 \%$ with $95 \%$ and an adjusted $\mathrm{Cl}(0.001325 \%$ to $0.011566 \%)$. Conclusions Our results indicate that as of mid-June 2020, seroprevalence in Palestine persists low and is inadequate to provide herd immunity, emphasising the need to maintain health measures to keep the outbreak under control. Population-based seroprevalence studies are to be conducted periodically to monitor the SARS-CoV-2 seroprevalence in Palestine and inform policymakers about the efficacy of their surveillance system.

\section{INTRODUCTION}

COVID-19 is an infectious respiratory disease caused by novel coronavirus SARS-CoV-2. ${ }^{1}$ Since its emergence in Wuhan, China in December 2019, ${ }^{2}$ SARS-CoV-2 has spread rapidly around the globe. A global pandemic was declared by the WHO, ${ }^{34}$ and new cases and deaths are being reported daily. ${ }^{5}$

Most authorities rely on PCR testing results to estimate the number of COVID-19

\section{Strengths and limitations of this study}

- The random selection of household residents collected between 15th June 2020 and 30th June 2020.

- Good representation of women and children among the 1136 participants from Medicare laboratory referrals between 1st May 2020 and 9th July 2020 .

- Low representation in women represented in the random sample.

- The random sample did not include children under the age of 15 .

cases and make up-to-date decisions. ${ }^{6}$ Thus, numbers of patients tested positive for SARS-CoV-2 through PCR testing, symptomatic patients, those admitted to hospitals or deceased from COVID-19 are updated on a daily basis. However, the data may exclude a fraction of the population with previous mild or asymptomatic COVID-19 that has not been tested by PCR. The proportion of the population who have overcome the infection without being noticed can probably be approximated by testing for antibodies against SARS-CoV-2. Antibodies may confer immunity to repeat infection and a high proportion of immune individuals can attenuate the epidemic. Measures of anti-SARS-CoV-2 seroprevalence can also be used to estimate the clinical impact of COVID-19. To this effect, several serological surveys of SARS-CoV-2 have been done worldwide..$^{7-16}$

There is lack of data on the percentage of undiagnosed Palestinian population with previous mild or asymptomatic COVID-19. Prevalence of COVID-19 infections among Palestinians residing in the West Bank remains unknown and many are concerned about this uncertainty. To this end, we conducted a population cross-sectional-based seroepidemiological study to assess the spread of SARS-CoV-2 throughout the West Bank. The study included 2491 individuals, designed to 


\begin{tabular}{lll} 
Table 1 & The PPS sampling algorithm \\
Location & $\begin{array}{l}\text { \# of households } \\
\text { in the location }\end{array}$ & Cumulative \\
\hline L1 & X1 & C1 $1=$ X1 \\
L2 & X2 & C2 $=$ X1 $1+$ X2 \\
L3 & X3 & C3 $=$ X1 $1+X 2+X 3$
\end{tabular}

\begin{tabular}{|c|c|c|}
\hline . & . & . \\
\hline . & . & . \\
\hline Lk-1 & $\mathrm{Xk}-1$ & $\mathrm{Ck}-1=\mathrm{X} 1+\mathrm{X} 2+\ldots+\mathrm{Xk}-1$ \\
\hline Lk & $\mathrm{Xk}$ & $\mathrm{Ck}=\mathrm{X} 1+\mathrm{X} 2+\ldots+\mathrm{Xk}$ \\
\hline
\end{tabular}

PPS, probability proportional to size.

be representative by cities (1355 from randomly selected households and 1136 from laboratory referrals). Elecsys Anti-SARS-CoV-2 testing was done on 2455 adequate serum samples. Here, we describe the study design and the results of the first wave of the study.

\section{METHODS}

\section{Study design and participants}

The study conducted is a cross-sectional serological testing study aimed to investigate seropositivity for SARS-CoV-2 in the non-institutionalised Palestinian population residing in the West Bank.

The study involved 1355 participants from 11 governorates, including 112 localities (online supplemental table 1). We used three-stage cluster sampling to select 1395 households. The cluster of households or census track is considered to be a geographical location that is comprised of approximately 100 households. The process for conducting cluster sampling was carried as follows: (1) selecting a cluster of households, (2) selecting 10 households randomly from each cluster and (3) selecting a person at random from the selected household. The clusters were selected using probability proportional to size (PPS) sampling (table 1).

To select the number of clusters within each population location: (1) we calculated the sampling interval which equals the total number of households divided by the total number of clusters needed to be selected by the sample say, for example $(\mathrm{m})$. So, the sampling interval $\mathrm{SI}=\mathrm{N} / \mathrm{m}$, where $\mathrm{N}$ is the total number of households; (2) selected a random number $\mathrm{R} 0$ between 0 and SI; (3) calculated Ri as $\mathrm{R} 0+\mathrm{i} \times \mathrm{SI}$, a cluster is selected in $\mathrm{Li}$ if $\mathrm{Ri}$ belongs to the interval (Ci-1, Ci).

Field work was carried out between 15th June 2020 and 30 th June 2020 by a team of registered nurses, laboratory technicians, nursing students and laboratory technician students from the Arab American University (AAUP) following standardised health protocols. ${ }^{17}$ The random sample did not include children under the age of 15 and suffered from a low representation in women.
The study also included 1136 participants from Medicare medical laboratories network referrals between 1st May 2020 and 9th July 2020 in 16 branches in the West Bank (online supplemental table 2).

Participants donated a blood sample for antibodies detection. Blood samples were centrifuged, and serum was separated, labelled and stored at $-20^{\circ} \mathrm{C}$ at AAUP laboratory until it was used.

\section{Detection of antibodies}

Serological tests for 2455 adequate serum samples were done using an immunoassay for the qualitative detection of antibodies against SARS-CoV-2 (Elecsys Anti-SARS-CoV-2) in human serum by using the Cobas Analyzer cobas e 411 (Roche). The assay uses a recombinant protein representing the nucleocapsid (N) antigen for the determination of antibodies against SARS-CoV-2 with testing time of $18 \mathrm{~min}$. The assay was reported to have a specificity of $100 \%$, while the overall sensitivity was $83.87 \%$, rising to $87.0 \%$ at 14 days after onset of symptoms, $87.7 \% 21$ days after and $100 \%$ more than 40 days after. ${ }^{18}$ We included six samples from recovered cases with detected SARS-CoV-2 antibodies as a positive control.

\section{Statistical analysis}

We estimated seroprevalence as the proportion of individuals who had a positive result in the total SARS-CoV-2 antibodies in the immunoassay.

We used Wilson Method With Continuity Correction and Boundary Truncation (WCCBT) to construct 95\% CI for the population parameter of seroprevalence. ${ }^{19}$

We calculated the adjusted CI. Since the specificity of the kit used for the antibodies test is $100 \%$ and $83 \%$, respectively, ${ }^{18}$ we adjusted the CI for seroprevalence according to the following transformation: adjusted lower confidence limit $=\max [0,\{\mathrm{a}-(1-\mathrm{sp})\} /(\mathrm{sp}+\mathrm{se}-1)]$ and adjusted upper confidence limit $=\min [1,\{\mathrm{~b}-(1-\mathrm{sp})\} /(\mathrm{sp}+\mathrm{se}-1)] .^{20}$ The low representation in women represented in the random sample required reweighting by gender. As for the Medicare labs sample, the obtained estimates from the lab data are considered to be unbiased and did not require reweighting.

\section{Patient and public involvement}

The study involved 1355 participants from 11 governorates, including 112 localities, and included 1136 participants from Medicare laboratory referrals. The development of the research question and outcome measures was based on the public priorities to know the seroprevalence in the Palestinian community. Participants were notified of the results of the tests.

\section{RESULTS}

Of the 1395 eligible individuals residing in households selected using three-stage cluster sampling, 1355 participants agreed to participate in the study. The proportion of women was lower compared with men (137 and 1218, 

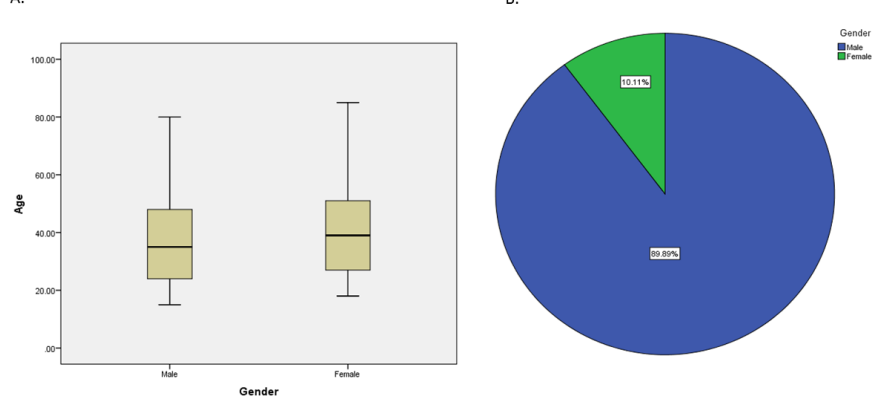

Figure 1 (A) Age and (B) gender distribution of the 1355 participants from Palestinian households in the West bank. Blood samples were collected between 15th June 2020 and 30th June from the participants.

respectively) including 349 in age group (15-24), 314 in age group (25-34), 377 in age group (35-49) and 315 in age group $(50+)$ (figure 1$)$. The majority of the participants did not report having symptoms in the last 3 months nor prevailing chronic diseases (online supplemental tables 3 and 4). None of the tested specimens revealed presence of antibodies against SARS-CoV-2. A 95\% CI for the population parameter of seroprevalence was $(0.0$ to $0.0036)$. A $95 \%$ CI for the population parameter of seroprevalence was $(0.0$ to 0.0036$)$, and an adjusted CI of (0 to 0.0043 ).

The study also included 1136 participants from Medicare laboratory referrals in 16 branches. The proportion of men was lower than women (395 and 741, respectively) including 71 in age group lower than 15, 173 in age group (15-24), 297 tests in age group (25-34), 290 in age group (35-49) and 305 in age group (50+) (figure 2). Records of symptoms and chronic diseases were unattainable. Out of the 1136 tested participants, three men, aged 38, 58, 59; and 1 woman, aged 40, revealed antibodies against SARS-CoV-2, with $95 \%$ CI (0.0011 to 0.0096) and an adjusted CI of (0.001325 to 0.011566$)$.

All serum samples were adequate for testing. Out of the 1136 tested participants, 4 revealed antibodies against SARS-CoV-2 with $95 \%$ CI (0.0011 to 0096).
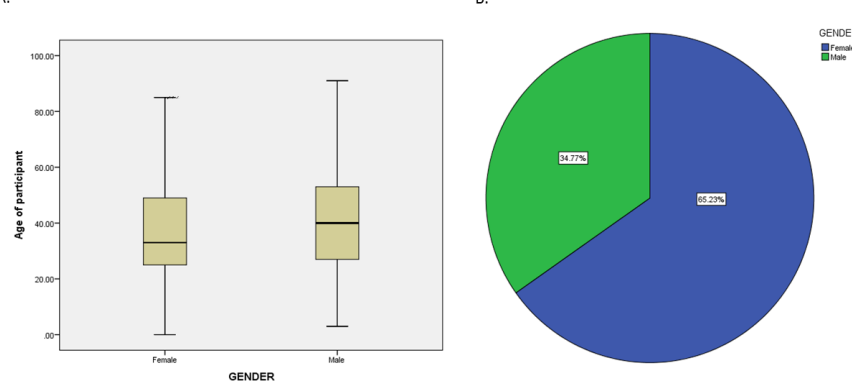

Figure 2 (A) Age and (B) gender distribution of the 1136 participants visiting Medicare laboratories between the 1st May 2020 and 9th July 2020 for a routine check-up.

\section{DISCUSSION}

To our knowledge, this is the first SARS-CoV-2 seroprevalence study in the Palestine. The findings from this seroprevalence study for SARS-CoV-2 indicate that the estimated seroprevalence of the total SARS-CoV-2 antibodies persists low as of mid of June. The random sample of Palestinians living in the West Bank yielded $0 \%$ seroprevalence with $95 \%$ and an adjusted CI of $(0 \%$ to $0.0043 \%$ ), while the lab referral samples yielded an estimated seroprevalence of $0.354 \%$ with $95 \%$ and an adjusted CI $(0.001325 \%$ to $0.011566 \%)$.

The low SARS-CoV-2 antibody prevalence in our population can be explained by the imposed total lockdown in the first 3 months when cases were under control and only reached around 800 cases by May. Our sampling dates ranged from 15th June 2020 to 30th June 2020 in the random sample and between 1st May 2020 and 9th July 2020 in the lab referral samples. The government of Palestine extended the state of emergency and imposed total lockdown in the first 3 months. The samples were collected after this strict lockdown which was accompanied by a drop in disease prevalence. COVID-19 cases were under control. The reported cases by the Palestinian Ministry of Health at the beginning of the data collection process by mid-June were 689 cases, and the reported cases rose to 2765 cases by the end of June. ${ }^{21}$ However, it takes 10-14days for antibodies against SARS-CoV-2 to go up, therefore, our results indeed reflect the situation by mid of June. Also, it was previously reported that antibodies against SARS-CoV-2 may drop or even disappear in patients with mild COVID-19, ${ }^{22-24}$ which may have led to the inability to detect antibodies in a few previously positive cases.

Seroprevalence in Palestine is very close to that identified in Jordan ( $0 \%$ prevalence),${ }^{15}$ a neighbouring country, which was explained to be due to the strict closures implemented by the Jordanian government and the eventual curtailing of infections in Jordan. Also, seroprevalence in Maine and Montana was $0.4 \%$ and $0.5 \%$, respectively, by end of August 2020. ${ }^{25}$ In comparison with other countries like Spain, Italy, Japan, India, Los Angeles, Germany and Switzerland, the seroprevalence in Palestine is low. ${ }^{7-16}$ Similarly, the seroprevalence among Palestinians residing in the West Bank was shown to be lower than that in Israel as reported in the first conducted serological study of 1700 tests. The study suggested that $2.5 \%$ of the Israeli population have had the coronavirus. ${ }^{26}$ It is, however, noteworthy that a comparison with other countries may be problematic due to the timing and the stage of the pandemic which may vary, affecting the seroprevalence estimates.

A key strength of our study is the random selection of household residents collected between 15th June 2020 and 30th June 2020. The clusters were selected using PPS sampling. This technique ensures getting self-weighting sample which can be used to produce unbiased estimators for the parameters of interest. The sample was supposed to be stratified by gender and age. However, 
the female response was low and samples from women were more difficult to obtain due to a cultural boundary. In fact, women were less open to welcoming a team of nurses at home for research purposes. Also, the random sample did not include children under the age of 15 to avoid anxiety due to fear of needles in the study to eliminate personal fears due to blood withdrawal. To ensure that the estimated prevalence in the random sample was unbiased, we reweighted the sample by gender. Except for cultural issues resulting in the low response rate from women in the random sample, we do not believe there were any issues that would lead participants to opt out from the study.

As for the lab referral samples, it represents the population of the lab referrals between the 1st May 2020 and 9 th July 2020. The seroprevalence in this sample was 4 positive cases out of the 1136 samples tested. Since the seroprevalence within the lab referral population is close to zero, we used WCCBT to construct a 95\% CI for the population parameter of seroprevalence $(0.0011$ to 0096). In comparison with household samples that were collected between 15th and 30th of June, laboratory referral samples include a good representation of women and children below the age of 15 (figure 2). Also, samples were collected starting from 1st May, which may better represent the small peaks of SARS-CoV-2-reported cases in Palestine and thus explain the presence of few positive samples in comparison with null in household samples. The sample was randomly selected by the lab data managers. Although women were not represented among the 1355 adults from Palestinian households, they were represented among the 1136 individuals visiting laboratories; out of the 1136,741 , more than $50 \%$ of the participants, were women (figure 2). Hence, the obtained estimates from the lab data are considered to be unbiased.

Our study only detected SARS-CoV-2 antibodies. It was previously reported that antibodies against SARS-CoV-2 may drop or even disappear in patients with mild COVID19 , which may have led to the inability to detect antibodies in a few previously positive cases. ${ }^{22}{ }^{23}$ Also, it is important to recognise that cellular immunity may play a role in providing immunity against SARS-CoV-2 reinfection. ${ }^{18}$ Further studies aimed at testing cellular immunity are important. It is also noteworthy that previous studies have indicated that asymptomatic individuals were reported to have a weaker immune response to SARS-CoV-2 infection and a higher percentage of asymptomatic individuals became seronegative when compared with symptomatic individuals in the early recovering phases. The reduction in neutralising antibody levels may have implications for immunity strategy and serological surveys. ${ }^{24} 27$

In conclusion, our study provides estimates of SARS-CoV-2 seroprevalence in Palestine. Our results reflect the seroprevalence as of mid of June. It is noteworthy that on 25th May, the government of Palestine eased the restrictions following a decline in cases which led to a surge in cases beginning of July, with the epicentre of the epidemic in Hebron accounting for over $70 \%$ of active cases implying a possible increase in seroprevalence after July. Reported cases rose to 2765 cases by the end of June. By December 2020, the cumulative number of positive cases grew to 150046 cases. ${ }^{21}$ In this situation, health measures have to be taken to keep the outbreak under control, in order to monitor the SARS-CoV-2 seroprevalence in Palestine and inform policymakers about the efficacy of their surveillance system. With the surge in cases, conducting population-based seroprevalence studies on a regular basis is important.

Acknowledgements We thank the participants for their cooperation. We thank the registered nurses, laboratory technicians, nursing students and laboratory technician students from the Arab American University: Ahmad Hodrub, Mohammad Faisal, Wajdi Tu'ma, Adam Maraw'a, Sharhabeel Nasrallah, Hisham Zahran and Mohammad Barakat. We thank Medicare labs for providing blood samples of individuals visiting their laboratories.

Contributors Research design-NQ, ZS and FA. Field work coordination-MA and IAK. Data analysis - FA. Manuscript writing - NQ. Manuscript revisions-NQ, FA and ZS. Methodology and lab work-NQ, KH, NB and HS.

Funding The authors have not declared a specific grant for this research from any funding agency in the public, commercial or not-for-profit sectors.

Competing interests None declared.

Patient consent for publication Not required.

Ethics approval Approval from the National Ethical Committee was obtained (PHRC/HC/737/20). Written informed consent was obtained from the 1355 study participants and approvals were obtained from Medicare laboratories for samples to be tested.

Provenance and peer review Not commissioned; externally peer reviewed.

Data availability statement Data are available upon reasonable request.

Supplemental material This content has been supplied by the author(s). It has not been vetted by BMJ Publishing Group Limited (BMJ) and may not have been peer-reviewed. Any opinions or recommendations discussed are solely those of the author(s) and are not endorsed by BMJ. BMJ disclaims all liability and responsibility arising from any reliance placed on the content. Where the content includes any translated material, BMJ does not warrant the accuracy and reliability of the translations (including but not limited to local regulations, clinical guidelines, terminology, drug names and drug dosages), and is not responsible for any error and/or omissions arising from translation and adaptation or otherwise.

Open access This is an open access article distributed in accordance with the Creative Commons Attribution Non Commercial (CC BY-NC 4.0) license, which permits others to distribute, remix, adapt, build upon this work non-commercially, and license their derivative works on different terms, provided the original work is properly cited, appropriate credit is given, any changes made indicated, and the use is non-commercial. See: http://creativecommons.org/licenses/by-nc/4.0/.

ORCID iD

Nouar Qutob http://orcid.org/0000-0003-2746-7367

\section{REFERENCES}

1 Mayo clinic. Coronavirus disease (COVID-19), 2019.

$2 \mathrm{Lu} \mathrm{H}$, Stratton CW, Tang Y-W. Outbreak of pneumonia of unknown etiology in Wuhan, China: the mystery and the miracle. J Med Virol 2020;92:401-2

3 World Health Organization. Novel coronavirus (2019-NCoV) situation report, 2020: 12.

4 Hui DS, I Azhar E, Madani TA, et al. The continuing 2019-nCoV epidemic threat of novel coronaviruses to global health - The latest 2019 novel coronavirus outbreak in Wuhan, China. Int J Infect Dis 2020;91:264-6.

5 COVID-19 Dashboard by the center for systems science and engineering (CSSE) at Johns Hopkins University (JHU).

6 Onder G, Rezza G, Brusaferro S. Case-Fatality rate and characteristics of patients dying in relation to COVID-19 in Italy. JAMA 2020;323:2019-20. 
7 Snoeck CJ, Vaillant M, Abdelrahman T. Prevalence of SARS-CoV-2 infection in the Luxembourgish population the CON-VINCE study. Medrxiv 2020:2020.05.11.20092916.

$8 \mathrm{Wu}$ X, Fu B, Chen L, et al. Serological tests facilitate identification of asymptomatic SARS-CoV-2 infection in Wuhan, China. J Med Virol 2020;92:2-3.

9 Bryan A, Pepper G, Wener MH, et al. Performance characteristics of the Abbott architect SARS-CoV-2 IgG assay and seroprevalence in Boise, Idaho. J Clin Microbiol 2020;58:e00941-20. doi:10.1128/ JCM.00941-20

10 Stringhini S, Wisniak A, Piumatti G. Repeated seroprevalence of anti-SARS-CoV-2 IgG antibodies in a population-based sample from Geneva, Switzerland. medRxiv 2020:2020.05.02.20088898

11 Valenti L, Bergna A, Pelusi S. SARS-CoV-2 seroprevalence trends in healthy blood donors during the COVID-19 Milan outbreak. medRxiv 2020:2020.05.11.20098442

12 Shakiba M, Hashemi Nazari SS, Mehrabian F, et al. Seroprevalence of COVID-19 virus infection in Guilan Province, Iran. medRxiv 2020:2020.04.26.20079244.

13 Pollán M, Pérez-Gómez B, Pastor-Barriuso R, et al. Prevalence of SARS-CoV-2 in Spain (ENE-COVID): a nationwide, population-based seroepidemiological study. Lancet 2020;396:535-44.

14 Stadlbauer D, Amanat F, Chromikova V, et al. SARS-CoV-2 seroconversion in humans: a detailed protocol for a serological assay, antigen production, and test setup. Curr Protoc Microbiol 2020;57:2425-7.

15 Sughayer MA, Mansour A, Al NA, et al. Covid-19 seroprevalence rate in healthy blood donors from a community under strict lockdown measures. medRxiv 2020:2020.06.06.20123919.

16 Sood N, Simon P, Ebner P, et al. Seroprevalence of SARS-CoV-2Specific antibodies among adults in Los Angeles County, California, on April 10-11, 2020. JAMA 2020;323:2425-7.
17 World Health Organization. Ethical standards for research during public health emergencies: distilling existing guidance to support COVID-19, 2020: 1-4.

18 Mahase E. Covid-19: Two antibody tests are "highly specific" but vary in sensitivity, evaluations find. BMJ 2020;369:m2066.

19 Sakthivel Sivam SM. Everything or Nothing - A Better Confidence Intervals for Binomial Proportion in Clinical Trial Data Analysis. Sakthivel Sivam, Quartesian LLC, Princeton, New Jersey Subbiah Meenakshisundaram, L. N Government College, Ponneri, India 2014;2016.

20 Diggle PJ. Estimating prevalence using an imperfect test. Epidemiol Res Int 2011:2011:1-5

21 Coronavirus (COVID-19) in Palestine. Available: https://corona.ps/

22 Bölke E, Matuschek C, Fischer JC. Loss of Anti-SARS-CoV-2 antibodies in mild Covid-19. N Engl J Med 2020;383:1694-8.

23 Ibarrondo FJ, Fulcher JA, Goodman-Meza D, et al. Rapid decay of Anti-SARS-CoV-2 antibodies in persons with mild Covid-19. N Engl $J$ Med 2020;383:1085-7.

24 Zhao J, Yuan Q, Wang H, et al. Antibody responses to SARS-CoV-2 in patients with novel coronavirus disease 2019. Clin Infect Dis 2020;71:2-3.

25 COVID-19 case surveillance public use data. Available: https://covid cdc.gov/covid-data-tracker/\#national-lab

26 Coronavirus herd immunity? Not in Israel, according to a serological study. Available: https://www.jpost.com/israel-news/coronavirusherd-immunity-not-in-israel-according-to-a-serological-study630059

27 Wu L-P, Wang N-C, Chang Y-H, et al. Duration of antibody responses after severe acute respiratory syndrome. Emerg Infect Dis 2007;13:1562-4. 